\title{
The Correlation Analysis of Influencing Factors and Harmfulness of Expressway Traffic Accident
}

\author{
Xiaoli Huang ${ }^{1}$, Yaolong Liu ${ }^{1}$, Jin Duan ${ }^{2}$, Huaming Zhang ${ }^{3}$ \\ 1College of Economics and Management, Taiyuan University of Technology, Taiyuan, 030600; \\ 2College of Politics and Law Institute, Taiyuan University of Technology, Taiyuan, 03600; \\ 3 Lightning Prevention and Observation Center of Shanxi Province, Taiyua, 030002)
}

\section{高速公路交通事故影响因素及危害性关联分析}

\author{
黄晓丽 ${ }^{1}$, 刘耀龙 ${ }^{1^{*}}$, 段锦 ${ }^{2}$, 张华明 $^{3}$ \\ ${ }^{1}$ 太原理工大学 经济管理学院, 太原, 030600; \\ 2 太原理工大学 政法学院, 太原, 030600; \\ ${ }^{3}$ 山西省雷电防护监测中心,太原, 030002
}

\begin{abstract}
Highway traffic accidents are characterized by high death rate, large economic losses and so on. Based on the gray system theory, the gray correlation analysis model of accident causes and hazard indexes is set up, with the number of accidents caused by different reasons as the standard data array and hazard indexes of traffic accident as the correlation data array, and the correlation degree of each factor to the accident risk is calculated. The results shows that the main reasons for the freeway traffic accident deaths for pedestrians and passengers occupied lane illegally. The main cause of injury and direct property loss is motor vehicle violations. Improving the driving people's traffic laws, enhancing the sense of safety of pedestrians can reduce the risk of highway traffic safety.
\end{abstract}

Keywords: highway accident; influence factor; harmfulness; correlation

\section{摘 要}

高速公路交通事故具有高致死率、经济损失大 等特点。本研究采用灰色关联分析法, 以不同

*通讯作者: liuyaolong@tyut.edu.cn.
原因造成的事故起数为比较数列, 以反映交通 事故危害性指标为参考数列, 建立事故因素与 危害性指标的关联模型, 计算各个因素对事故 危害性的关联度。结果表明: 高速公路事故人 员死亡的主要致因为行人及乘车人违法占道; 造成人员受伤和直接财产损失的主要原因为 机动车违法行为。提高驾驶人的交通法律素 养, 提升行人的交通安全意识是降低高速公路 道路交通安全风险的有效手段。

关键字: 高速公路事故; 影响因素; 危害性; 关联度

\section{1. 引言}

道路交通事故是一个全球性的公共卫生 与安全问题。据 WHO 统计, 每年全球约有 120 万人死于道路交通事故, 2000-5000 万人因道 路交通事故遭受非致命性伤害。据估算, 到 2030 年由于道路交通事故导致的死亡将上升 至人类各种死亡因素的第五位, 到时每年约有 240 万人死于道路交通事故[1]。近年来, 我国 高速公路建设发展迅速, 公路里程由 2005 年 的 4.1 万公里上升至 2014 的 11.19 万公里, 年 增速达 $7.43 \%$ 。随着高速公路快速建设, 道路 交通事故也逐年增长。1995 年我国高速公路 交通事故数为 4590 起[2], 2013 年上升至 8693 起, 年增长率超过 $3.6 \%$ 。其高致死率和经济 损失巨大使得高速公路交通安全问题受到学 


\section{Risk Analysis and Crisis Response in Big Data Era (RAC-16)}

术界和政府部门的高度关注。

目前, 国外学者对高速公路事故研究主要 包括事故风险点研究[3-5]和交通事故原因分 析[6]。前者多运用事故风险指数、地质统计学 等方法, 识别交通事故易发点; 后者则是通过 对事故历史数据的挖掘, 探讨交通事故的原因 及对策。国内学者则关注不同环境和情形中高 速公路事故特点 (特征、变化规律、时空分布) [7-11]以及恶劣天气条件对高速公路交通事故 的影响[12-16]。此外, 亦有不少学者进行高速 公路交通事故数预测的研究[17-21]。明确风险 来源才能对更好地对风险进行管理[22-24]。本 研究针对由人、车、道路和环境组成的道路交 通事故复杂系统, 从高速公路交通事故危害性 和致险因素视角出发, 运用灰色关联模型定量 评价各项因素的危害性关联程度, 进而提出降 低事故综合风险的对策建议, 供政府及相关部 门制定安全管理和预防措施参考。

\section{2. 数据来源和研究方法}

\section{1 数据来源}

高速公路交通事故危害性采用事故起数、 死亡人数、受伤人数、直接财产损失和当量死 亡人数五项指标加以表征。其中, 事故起数反 映交通事故的数量特征, 死亡人数、受伤人数、 直接财产损失和当量死亡人数从不同侧面反 映交通事故的严重程度。当量死亡人数是死亡 人数、受伤人数和直接财产损失的综合指标, 其计算方法参见文献[25]。

高速公路交通事故的影响因素分为机动 车违法、机动车非违法过错、非机动车违法、 行人乘车人违法、道路和意外六个类型。其中, 机动车违法行为主要包括: 超速行驶、酒后行 驶、逆向形式等; 机动车非违法过错则包括: 制动不当、转向不当、油门控制不当等; 非机 动车违法行为包括: 逆向行驶、违法抢行、违 法占道行驶等; 行人乘车人违法包括: 违法上 道路行驶、违法占道、违反交通信号等; 道路 原因多由于道路缺陷或未设置安全标识等, 意 外原因是由于天气或其他状况造成的事故。

本研究样本数据为 2009-2013 年我国高速 公路各影响因素造成的交通事故起数、死亡人 数、受伤人数和直接财产损失。数据来源于《中 华人民共和国道路交通事故统计年报》。

\section{2 研究方法}

本文运用灰色关联分析法来衡量事故诱 因对于高速公路交通事故危害性的关联程度, 即以不同成因的事故起数为比较数列, 以反映 交通事故危害性指标为参考数列, 建立事故因 素与危害性指标的关联模型, 进而计算各影响 因素对于事故危害性的关联度。

具体计算步骤如下:

（1）确定参考序列和比较序列 本文选取可 以反映各年份全国高速公路交通严重程度的 指标 (死亡人数、受伤人数、直接财产损失、 当量死亡人数) 作为参考数列, 记为:

$Y_{i}=\left\{Y_{i}(t) \mid \mathrm{t}=1,2, \ldots, m ; i=1,2 \ldots, n\right\} \quad$ (1) 式中, $Y_{i}(t)$ 表示第 $\mathrm{t}$ 个样本中的第 $\mathrm{i}$ 个危害性 指标的值; $\mathrm{m}$ 表示参考数列样本总数, $\mathrm{n}$ 表示 危害性指标的个数。将各年份不同影响因素造 成的事故起数作为比较数列, 记为:

$X_{j}=\left\{X_{j}(t) \mid \mathrm{t}=1,2, \ldots, \quad m ; j=1,2, \ldots, \mathrm{k}\right\}$

式中, $X_{i}(t)$ 表示第 $\mathrm{j}$ 项因素在第 $\mathrm{t}$ 个样本中的 值; $\mathrm{k}$ 表示因素个数。

（2）数据无量纲化处理 由于各指标原始数 据量纲不同, 不能直接对其进行数据分分析, 需对原始数据进行规范化处理, 常用的方法 有: 初值法、均值法、标准化法和极差法, 而 灰色关联度的分析采用标准化处理结果比较 切合实际 [26], 故而本文采用标准化法对数据 进行无量纲化处理:

$$
\begin{gathered}
Y_{i}^{\prime}(t)=\left[Y_{i}(t)-\bar{Y}_{i}\right] / S_{i} \\
X_{j}^{\prime}(t)=\left[X_{j}(t)-\bar{X}_{j}\right] / S_{j}
\end{gathered}
$$

式中, $Y_{i}^{\prime}(t)$ 和 $X_{j}^{\prime}(t)$ 为数据规范化结果; $\bar{Y}_{i} 、 \bar{X}_{j}$ 分 别为第 $\mathrm{i}$ 项参考数列和第 $\mathrm{j}$ 项因素事故数的均 值; $S_{i} 、 S_{j}$ 分别为第 $\mathrm{i}$ 项参考数列和第 $\mathrm{j}$ 项因素 事故数的标准差。

(3) 关联系数和关联度计算 对无量纲数据 求关联系数的公式如下:

$$
\begin{aligned}
\xi_{i j}(t) & =\frac{\Delta_{\min }+\eta \Delta_{\max }}{\Delta_{i j}(t)+\eta \Delta_{\max }} \\
\text { 式中, } \Delta_{i j}(t) & =\left|X_{j}^{\prime}(t)-Y_{i}^{\prime}(t)\right|, \\
\Delta_{\min } & =\min _{i} \min _{j} \min _{t} \Delta_{i j}(t),
\end{aligned}
$$




$$
\Delta_{\max }=\max _{i} \max _{j} \max _{t} \Delta_{i j}(t)
$$

和 $\eta$ 为分辨系数, 且 $\eta \in[0,1]$, 一般取 0.5 。第 $\mathrm{i}$ 项参考数列与第 $\mathrm{j}$ 项因素事故数的关联度计算 采用均值法:

$$
r_{i j}=\frac{1}{m} \sum_{t=1}^{m} \xi_{i j}(t)
$$

式中, $r_{i j}$ 和 $\xi_{i j}$ 分别为第 $\mathrm{i}$ 项参考数列与第 $\mathrm{j}$ 项因 素的关联度和关联系数。

\section{3. 研究结果}

2009-2013 年, 我国高速公路交通事故起数、 死亡人数、受伤人数、直接财产损失及当量死 亡人数统计结果见表 1 。我国高速公路交通事 故危害性呈现出波动下降趋势（图 1)。

\section{表 1 高速公路交通事故统计数据}

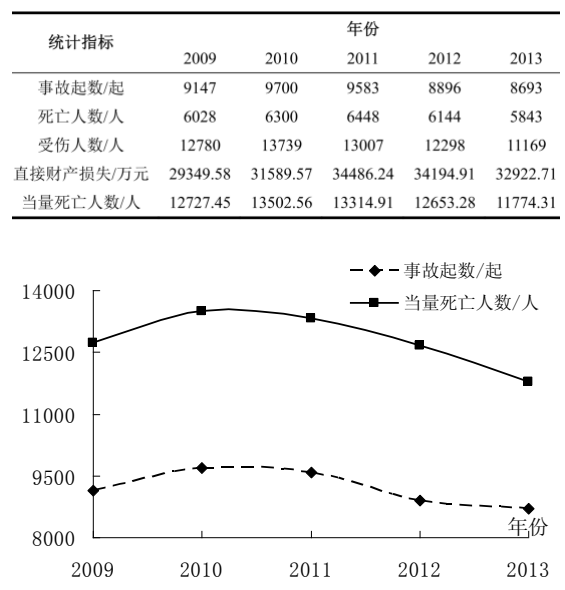

图 1 高速公路事故数和当量死亡人数趋势

高速公路各影响因素造成的交通事故起 数统计结果见表 2 。可以看出, 机动车违法行 为导致的事故数占总数的 $80 \%$, 是造成高速公 路道路交通事故的主要原因; 机动车非违法过 错和行人乘车人违法是次要原因, 事故比分别 为 $10 \%$ 和 $9 \%$ 。

采用公式 (1) - (7), 计算出不同事故 原因与高速公路交通事故危害性的关联度值 （表 3)。按照 Likert 五点态度量表方法, 将 关联度划分为 5 等级, 即关联度 $<0.2$, 极低关 联性; $0.2 \leqslant$ 关联度 $<0.4$, 低关联性; $0.4 \leqslant$ 关联度 $<0.6$, 中关联性; $0.6 \leqslant$ 关联度 $<0.8$,
高关联性; 关联度 $\geqslant 0.8$, 极高关联性。可以看 出, 与高速公路交通事故人员死亡关联性极高 的因素有五个, 分别为人违法占道 (行人及乘 车人违法行为) 、违法装载、超速行驶、违法 占道行驶 (均为机动车违法行为) 和油门控制 不当 (机动车非违法行为), 其关联度数值均 大于 0.8 。此五项因素易造成严重交通事故, 应引起相关部门重视。

\begin{tabular}{|c|c|c|c|c|c|c|}
\hline \multirow{2}{*}{ 事故原因分类 } & \multirow{2}{*}{ 事故原因细分 } & \multicolumn{5}{|c|}{ 年份 } \\
\hline & & 2009 & 2010 & 2011 & 2012 & 2013 \\
\hline \multirow{21}{*}{$\begin{array}{l}\text { 机动车 } \\
\text { 违法行为 }\end{array}$} & 超速行驶 & 795 & 893 & 889 & 772 & 395 \\
\hline & 酒后驾驶 & 92 & 58 & 95 & 119 & 120 \\
\hline & 逆向行驶 & 72 & 91 & 80 & 71 & 55 \\
\hline & 疲劳驾驶 & 921 & 827 & 887 & 681 & 704 \\
\hline & 违法变更车道 & 223 & 308 & 251 & 240 & 247 \\
\hline & 违法超车 & 124 & 161 & 132 & 79 & 62 \\
\hline & 违法倒车 & 38 & 41 & 56 & 43 & 47 \\
\hline & 违法掉头 & 53 & 67 & 58 & 36 & 38 \\
\hline & 违法会车 & 27 & 29 & 23 & 3 & 8 \\
\hline & 违法牵引 & 5 & 4 & 6 & 1 & 0 \\
\hline & 违法抢行 & 10 & 7 & 13 & 12 & 20 \\
\hline & 违法上道路行驶 & 264 & 251 & 236 & 290 & 300 \\
\hline & 违法停车 & 125 & 109 & 94 & 76 & 60 \\
\hline & 违法占道行驶 & 109 & 136 & 119 & 121 & 96 \\
\hline & 违法装载 & 106 & 108 & 108 & 102 & 89 \\
\hline & $\begin{array}{l}\text { 违法装载超限 } \\
\text { 及危险品运输 }\end{array}$ & 1 & 1 & 1 & 0 & 6 \\
\hline & 违反交通信号 & 42 & 50 & 64 & 52 & 48 \\
\hline & 未按规定让行 & 61 & 51 & 43 & 32 & 29 \\
\hline & 无证驾驶 & 110 & 106 & 145 & 129 & 122 \\
\hline & 不按规定使用灯光 & 15 & 15 & 23 & 7 & 7 \\
\hline & 其他影响安全行为 & 4422 & 4555 & 4560 & 4266 & 4477 \\
\hline \multirow{4}{*}{$\begin{array}{c}\text { 机动车 } \\
\text { 非违法行为 }\end{array}$} & 制动不当 & 62 & 106 & 94 & 71 & 70 \\
\hline & 转向不当 & 65 & 64 & 61 & 78 & 62 \\
\hline & 油门控制不当 & 3 & 7 & 5 & 3 & 1 \\
\hline & 其他操作不当 & 686 & 845 & 700 & 779 & 737 \\
\hline \multirow{7}{*}{$\begin{array}{l}\text { 非机动车 } \\
\text { 违法行为 }\end{array}$} & 逆向行驶 & 2 & 2 & 6 & 2 & 5 \\
\hline & 违法抢行 & 0 & 0 & 0 & 1 & 1 \\
\hline & 违法上道路行驶 & 12 & 15 & 12 & 15 & 19 \\
\hline & 违法占道行驶 & 0 & 0 & 1 & 1 & 2 \\
\hline & 违反交通信号 & 0 & 1 & 0 & 0 & 2 \\
\hline & 未按规定让行 & 2 & 0 & 2 & 1 & 3 \\
\hline & 其他影响安全行为 & 0 & 1 & 3 & 2 & 3 \\
\hline & 违法上道路行驶 & 522 & 598 & 641 & 659 & 725 \\
\hline 行人及乘车人 & 人违法占道 & 1 & 1 & 3 & 2 & 0 \\
\hline \multirow[t]{2}{*}{ 违法行为 } & 违反交通信号 & 23 & 12 & 18 & 28 & 17 \\
\hline & 其他影响安全行为 & 63 & 102 & 82 & 52 & 61 \\
\hline 道路 & 道路 & 1 & 1 & 2 & 0 & 3 \\
\hline 意外 & 意外 & 90 & 77 & 70 & 70 & 52 \\
\hline
\end{tabular}

\section{表 2 高速公路事故成因统计数据}

与高速公路交通事故人员受伤关联性极 高的因素有七个, 分别为逆向行驶、违法超车 (均为机动车违法行为) 、油门控制不当 (机 动车非违法行为) 、违法掉头、超速行驶、违 法装载、违法占道行驶 (均为机动车违法行 为）。其中, 逆向行驶和违法超车事故起数与 


\section{Risk Analysis and Crisis Response in Big Data Era (RAC-16)}

受伤人数的关联度分别为 0.9321 和 0.9029 , 是造成人员受伤的首要因素。其他五个因素的 关联度亦超过 0.8 。这七项因素大部分为人为 因素, 其与违法超载相比, 伤亡率较轻, 但由 于这些因素造成的事故绝对数较多, 仍然需要 引起重视; 造成直接财产损失方面, 机动车违 法行为中的其他影响安全行为是最主要的诱 因, 其关联度为 0.8402 ; 其他关联性较高的因 素有: 违法上道路行驶、违反交通信号 (均为 行人及乘车人违法行为) 、违法倒车和无证驾 驶 (均为机动车违法行为)。

\section{表 3 事故原因与危害性指标关联度}

\begin{tabular}{|c|c|c|c|c|c|}
\hline & 事故原因 & 死亡人数 & 受彷人数 & 直接财产损失 & 当量死亡人数 \\
\hline \multirow{21}{*}{$\begin{array}{l}\text { 机动车 } \\
\text { 违法行为 }\end{array}$} & 超速行戨 & 0.8112 & 0.8595 & 0.5981 & 0.8676 \\
\hline & 酒后驾驶 & 0.5614 & 0.5882 & 0.6646 & 0.6010 \\
\hline & 逆向行使 & 0.7977 & 0.9321 & 0.5596 & 0.9293 \\
\hline & 疲劳鹄驶 & 0.6832 & 0.7023 & 0.5904 & 0.7244 \\
\hline & 速法变更车道 & 0.6576 & 0.7243 & 0.6113 & 0.6950 \\
\hline & 违法赫车 & 0.7303 & 0.9029 & 0.5339 & 0.8318 \\
\hline & 进法倒车 & 0.7007 & 0.6227 & 0.7795 & 0.6501 \\
\hline & 进法掉头 & 0.6957 & 0.8636 & 0.5456 & 0.8054 \\
\hline & 进法会车 & 0.6693 & 0.7917 & 0.5286 & 0.7489 \\
\hline & 进法䇦引 & 0.7773 & 0.7505 & 0.5910 & 0.7570 \\
\hline & 进法拖行 & 0.6684 & 0.6385 & 0.6120 & 0.6466 \\
\hline & 速法上道路行驶 & 0.5519 & 0.5538 & 0.6803 & 0.5811 \\
\hline & 违法停车 & 0.7418 & 0.7757 & 0.5089 & 0.7415 \\
\hline & 违法占道行致 & 0.8065 & 0.8312 & 0.5891 & 0.8156 \\
\hline & 违法裴裁 & 0.8178 & 0.8553 & 0.5825 & 0.8690 \\
\hline & $\begin{array}{l}\text { 速法装轹超限及 } \\
\text { 危险品运输 }\end{array}$ & 0.6020 & 0.6171 & 0.6003 & 0.6203 \\
\hline & 违反交通信号 & 0.7503 & 0.6176 & 0.7477 & 0.6597 \\
\hline & 未挍规定让行 & 0.6981 & 0.7320 & 0.5073 & 0.7010 \\
\hline & 无证驾驶 & 0.7078 & 0.5768 & 0.7611 & 0.6197 \\
\hline & 不按规定使用奵光 & 0.7597 & 0.7401 & 0.5809 & 0.7300 \\
\hline & 其他影响安全行为 & 0.7107 & 0.6896 & 0.6838 & 0.7539 \\
\hline \multirow{4}{*}{$\begin{array}{l}\text { 机动车非 } \\
\text { 违法行为 }\end{array}$} & 制动不当 & 0.7236 & 0.7738 & 0.6515 & 0.7871 \\
\hline & 转向不当 & 0.6007 & 0.6061 & 0.6334 & 0.6291 \\
\hline & 油门政制不当 & 0.8028 & 0.8867 & 0.5702 & 0.8682 \\
\hline & 其他㙅作不当 & 0.6365 & 0.6507 & 0.6490 & 0.6447 \\
\hline \multirow{7}{*}{$\begin{array}{l}\text { 非机动车 } \\
\text { 违法行为 }\end{array}$} & 逆向行哏 & 0.6981 & 0.5940 & 0.6967 & 0.6258 \\
\hline & 违法掩行 & 0.5638 & 0.5226 & 0.6841 & 0.5337 \\
\hline & 违法上道路行歌 & 0.6460 & 0.5796 & 0.6177 & 0.5977 \\
\hline & 速法占道行㜔 & 0.6171 & 0.6056 & 0.6839 & 0.6017 \\
\hline & 进反交通信号 & 0.6625 & 0.6232 & 0.5504 & 0.6274 \\
\hline & 未挍规定让行 & 0.5664 & 0.7045 & 0.5738 & 0.6396 \\
\hline & 其他影啝安全行为 & 0.6632 & 0.5882 & 0.8402 & 0.6397 \\
\hline \multirow{4}{*}{$\begin{array}{c}\text { 行人及乘 } \\
\text { 车人违法 } \\
\text { 行为 }\end{array}$} & 速法上道路行驶 & 0.5933 & 0.5881 & 0.7948 & 0.5829 \\
\hline & 违法占道 & 0.8196 & 0.6837 & 0.7266 & 0.7355 \\
\hline & 违反交通信号 & 0.5391 & 0.6048 & 0.6066 & 0.5717 \\
\hline & 其他影响安全行为 & 0.7083 & 0.7740 & 0.5790 & 0.7553 \\
\hline 道路 & 道路 & 0.6220 & 0.6439 & 0.6529 & 0.6517 \\
\hline 意外 & 意外 & 0.7420 & 0.7754 & 0.5285 & 0.7627 \\
\hline
\end{tabular}

与当量死亡人员关联性极高的因素有七 个, 逆向行驶 (关联度为 0.9293 ) 、违法装载 （关联度为 0.8690 ）、油门控制不当（关联度 为 0.8682 ) 、超速行驶 (关联度为 0.8676 )、 违法超车 (关联度为 0.8318 ) 、违法占道行驶
(关联度为 0.8156 ) 和违法掉头 (关联度为 0.8054）。以上因素中, 仅油门控制不当为机 动车非违法行为, 其他均为机动车违法行为。 可见, 就高速公路交通事故综合危害指标而 言, 机动车违法行为是首要因素。此外, 机动 车违法上道路行驶、非机动车违法上道路行 驶、行人及乘车人违法上道路行驶、违反交通 信号和违法抢行的关联度均小于 0.6 , 其导致 事故综合死亡风险的概率较低。

\section{4. 结论}

（1）道路交通事故的随机性和模糊性决定了 灰色关联模型的可适用性。本研究计算不同事 故成因对高速公路交通事故危害性的关联度 数值, 定量评价事故致险的的优先度, 基于数 据挖掘揭示高速公路事故影响因素的重要程 度, 具有一定的应用价值。特别是将该模型用 于不同路段定量评价该路段事故主要原因, 为 有关部门制定有针对性的安全管理对策提供 参考。

（2）造成我国高速公路事故人员死亡的最重 要因素是行人及乘车人违法占道。相比于机动 车违法行为、道路和意外因素, 行人及乘车人 的道路交通安全意识和法制意识成为制约我 国道路交通安全管理的主要因素。与人员受伤 关联性较高的因素有逆向行驶、违法超车, 而 机动车违法行为也是造成直接财产损失的主 要原因。

（3）当量死亡人数反映了高速公路交通事故 的综合危害程度。机动车违法行为 (如逆向行 驶、违法装载、超速行驶、违法超车、违法占 道行驶和违法掉头) 是造成我国高速公路交通 事故综合风险的主要原因。建议道路交通安全 管理部门在进行事故风险管控中侧重驾驶人 交通规则、驾驶技能和法律素养方面的培训与 考查, 同时, 广泛关注行人和非机动车驾驶员 的交通安全意识和法律知识普及, 以此提高高 速公路道路交通安全管理水平。

\section{致谢}

本研究得到国家自然科学基金青年项目 (No.41301580); 太原理工大学校青年基金 (2015QN086); 太原理工大学引进人才科研启 动项目(TYUT-RC201110A)的资助。 


\section{参考文献}

[1] 陈冯富珍.全球道路安全现状报告.日内瓦: 世界卫生组织,2009: 1-44.

[2] 刘东,马社强,牛学军.我国高速公路交通 事故特点分析.中国人民公安大学学报:自 然科学版,2008, 4(4): 65-68.

[3] Kang S, Lee S M. Introducing Alignment Based Risk Indices into the Highway Traffic Accident Analysis. International Workshop on Computing in Civil Engineering, 2014: 465-477.

[4] Molla $\mathrm{M}$ M, Stone $\mathrm{M}$ L, Lee $\mathrm{E}$. Geostatistical Approach to Detect Traffic Accident Hot Spots and Clusters in North Dakota. Crash Data, 2014: 1-20.

[5] Vijaya R B, Sreedhar M. Evaluation of Reliability and Traffic Accident Frequency Rate by Using System Reliability Model-A Simulation Approach. International Journal of Statistics \& Analysis, 2013, 1(3): 11-20.

[6] Malvadkar P, Kawitkar R, Malvadkar P. Highway traffic accidents: a study on safety and development. International Journal of Electronics Communication \& Instrumentation Engineering Research \& Development, 2013, 3: 113-116.

[7] 胡思涛, 项乔君. 高速公路爬坡路段交通事 故特征分析.公路, 2013,5: 115-118.

[8] 陈定秋,朱德林. 高速公路交通事故的季节 变动规律分析及预测. 中国安全生产科学 技术,2013,3(9): 188-192.

[9] 李志斌,王炜,李晓伟等.高速公路车辆排 队尾部交通事故时空分布特征.交通运输 工程学报,2014,4: 76-81.

[10] 马壮林, 张宏璐, 谭晓伟. 高速公路连续下 坡路段交通事故时空分布特征研究.中国 安全科学学报,2014,5(24): 85-91.

[11] 马壮林,邵春福,胡大伟,等.高速公路交通 事故起数时空分析模型. 交通运输工程学 报,2012,2(12): 93-99.

[12] 潘娅英,朱占云, 沈萍月等.浙江省高速公 路交通事故特征及不良天气条件影响分 析.公路,2013,12: 157-160.

[13] 李迅, 甘璐,丁德平等.G2 京津塘高速公路
交通气象安全指数的预报研究.气象, 201,4(40): 466-472.

[14] 靖连天. 非极端恶劣气象条件与高速公路 交通事故之间的关系研究. 公路,2015,1: 114-119.

[15] 潘娅英, 朱占云, 沈萍月等. 浙江省高速公 路交通事故气象影响评价方法研究与应 用.公路,2015,6: 136-141.

[16] 张金满,谭桂容, 李飞等. 冰雪天气公路通 行条件预警指标.气象科技,2016,2(44): 331-335.

[17] Park S H, Kim S M, Ha Y G. Highway traffic accident prediction using VDS big data analysis. Journal of Supercomputing, 2016: 1-17.

[18] 崔立志. 高速公路交通事故的灰色预测模 型. 科学技术与工程, 2012, 12(19): 4843-4846.

[19] 孟祥海,郑来, 史永义. 考虑车道变换影响 的高速公路交通事故预测模型研究. 公路 交通科技,2014,6(31): 121-126.

[20] 王迎, 周燕. 基于广义线性模型的高速公路 交通事故预测. 公路工程, 2015,5(40): 115-119.

[21] 曹仁霞, 王迎, 马岳. 高速公路交通事故预 测研究.中外公路,2015,4: 326-329.

[22] Daniel Baloi. Risk Analysis Techniques in Construction Engineering Projects. Journal of Risk Analysis and Crisis Response, 2012, 2(2): 115-123.

[23] Dean K, Jacob K.A New Risk Management Model. Journal of Risk Analysis and Crisis Response,2012,2(4): 233-251.

[24] Seda U, Cengiz K. Fuzzy multicriteria risk assessment for hazardous waste management: the case of Istanbul. Journal of Risk Analysis and Crisis Response, 2011, 1(1): 29-41.

[25] 曹建军. 道路交通事故当量死亡人数的计 算模型研究.重庆交通大学学报(自然科 学版),2013,1(32): 91-95.

[26] 周化如,吕晓刚,郝海荣等.不同数据变换 方法对灰色关联度分析结果的影响.种子 科技,2005,5(23): 280-283. 